\title{
Radio Observations of Supernova Remnants in the M82 Starburst
}

\author{
Alan Pedlar, Tom Muxlow and Jon Riley \\ Jodrell Bank Observatory, University of Manchester, Cheshire SK11 9DL, UK; \\ ap@jb.man .ac.uk
}

Summary. We report on recent MERLIN, VLA and VLBI observations of the compact radio sources in the nearby starburst M82, with angular resolutions ranging from arcseconds to milliarcseconds. The spectral properties of the compact sources have been investigated by $15 \mathrm{GHz}$ VLA-Pie Town observations which show that 16 of the less luminous compact sources are, in fact, HII regions. However the steep non-thermal spectrum, parsec size and small variability of the remaining sources is consistent with their being supernova remnants. Several show clear shell structures at MERLIN resolution ( $\sim 50$ mas) and 5 have been resolved further using VLBI. Measurements of the most compact source $(41.95+575)$ shows an expansion velocity of $\sim 2000 \mathrm{~km} \mathrm{~s}^{-1}$, and one of the best defined SNR shells $(43.31+592)$ shows an expansion velocity of $\sim 10^{4} \mathrm{~km} \mathrm{~s}^{-1}$. Recent VLBI and MERLIN measurements confirm this expansion velocity and show little evidence for deceleration. We comment on the discrepancy between this measured expansion velocity and the low expansion velocities predicted theoretically for remnants in M82.

\section{Introduction}

Although studies of extragalactic supernova remnants (SNR) are currently limited by sensitivity, and to some extent angular resolution, such studies provide unique insights into the early evolution of radio supernovae and supernova remnants. Whilst the extensive studies of remnants in our own galaxy are particularly valuable for testing details of the physical processes occurring in individual remnants, they are limited by the fact that the youngest known galactic supernova remnant is over 300 years old. In addition the distances of many galactic remnants are quite uncertain, which has resulted in studies involving surface brightness-diameter plots being highly suspect. Furthermore because of the large differences in distance, statistical inferences based on observations with constant angular resolution and sensitivity are severely affected by selection effects.

Studies of extragalactic SNR in starburst galaxies have a number of advantages. Firstly the high star formation rate results in a relatively large number of young supernova remnants with ages measured in decades, rather than centuries. Secondly as the starburst region is typically a kiloparsec in extent, and as distances to even the nearest starburst galaxies are a few $\mathrm{Mpc}$, the relative distances to each $\mathrm{SNR}$ will only vary by $\sim 0.1 \%$. Finally 
radio synthesis techniques ensure that all the SNR are observed with same angular resolution and sensitivity. Hence as all the SNR are essentially at the same distance this corresponds to a constant linear resolution and surface luminosity limit.

\section{The Compact Sources in Messier 82}

Although radio supernova remnant studies have been carried out on several nearby starburst galaxies, by far the most extensive have been carried out in the Messier 82 starburst. Compact sources were first noted in M82 by Kronberg \& Wilkinson [5], but it was not until the advent of MERLIN [13] and the VLA [6] that $\sim 50$ compact radio sources were separated from the extended background. Although initially it was thought that some of these may be radio supernovae, the relatively low star formation rate inferred from FIR observations (see below), together with the lack of significant variability of most sources, implied that the more luminous compact sources were supernova remnants. This was subsequently confirmed by $5 \mathrm{GHz}$ MERLIN observations [10] in which all the sources were shown to be extended with sizes of typically a few parsecs and many had the shell or partial shell structures typical of remnants.

\subsection{Distinguishing Between Supernova Remnants and HII Regions}

The majority of the more luminous compact sources in M82 showed a steep spectrum, consistent with synchrotron emission from supernova remnants (e.g., [1]). Although from these initial spectral studies it appeared that only one compact source had a spectrum consistent with thermal emission, the sample only included about half $(\sim 26)$ of the known compact sources and as they were selected at relatively low frequencies hence had a bias towards non-thermal sources.

However recent work [9] determined the two point (5 and $15 \mathrm{GHz}$ ) spectra of 46 the compact sources in M82 by using the extended VLA (i.e., including the Pie-Town Antenna) at $15 \mathrm{GHz}$ which resulted in an angular resolution of 80 mas $(=1.2 \mathrm{pc}$ ) which can be directly compared to MERLIN $5 \mathrm{GHz}$ observations with 50 mas resolution. Fig. 1 shows a plot of spectral index against average brightness temperature of the compact sources, from which it can be seen that 16 had inverted spectra and brightness temperatures consistent with optically thick compact HII regions with emission measures of $\sim 10^{7} \mathrm{pc} \mathrm{cm}^{-6}$ implying relatively high thermal pressures. 


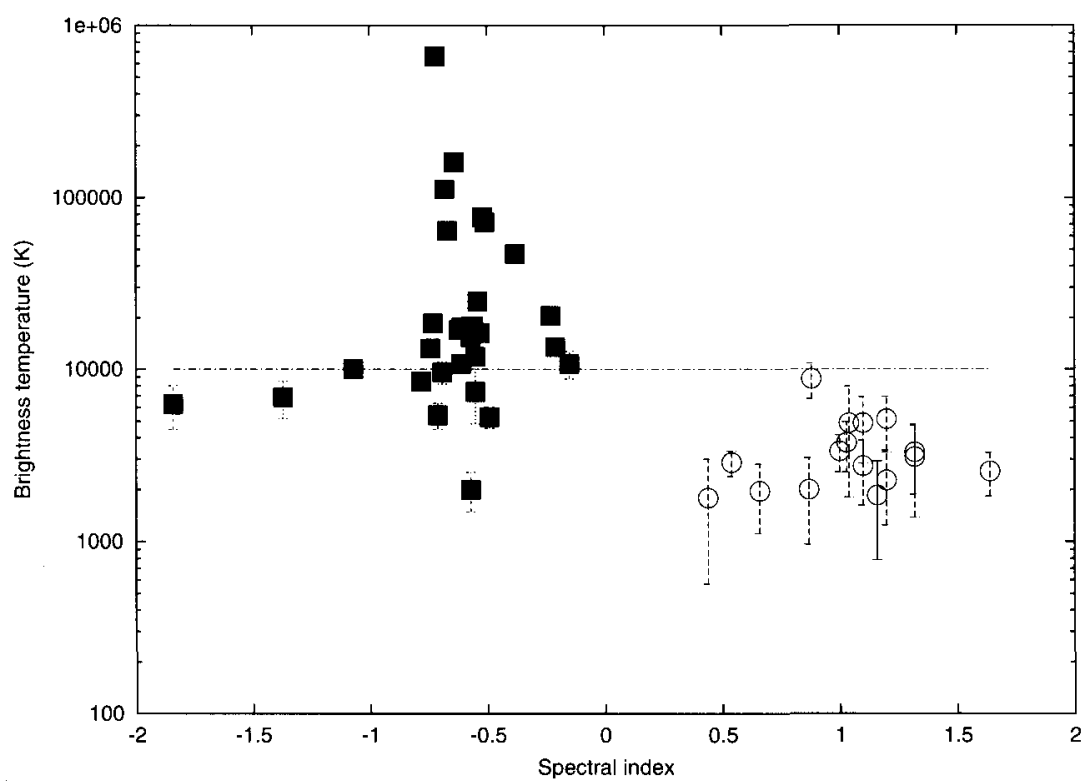

Fig. 1. The $5 / 15 \mathrm{GHz}$ spectral index plotted against the average brightness temperature of 46 of the compact sources in Messier 82 . The filled squares are consistent with supernova remnants, and the open circles with compact HII regions (adapted from $[9])$.

\section{Star Formation and Supernova Rates in M82}

The global star formation rate (SFR) of a galaxy can be estimated using a measurements in the ultra-violet, optical, infra-red and radio [4]. However as starburst galaxies usually contain large quantities of dust, the estimates of SFR from ultraviolet and optical data are uncertain due to dust extinction. M82 is no exception with $A_{v}$ typically 20-30 magnitudes. Fortunately the radio method [4] is not affected by dust extinction and hence the star formation rate can be estimated from the equation

$$
\operatorname{SFR}\left(M \geq 5 M_{\odot}\right)=\frac{L_{1.4}}{4.0 \times 10^{21}} \mathrm{M}_{\odot} \mathrm{yr}^{-1}
$$

where $L_{1.4}$ is the total $1.4 \mathrm{GHz}$ luminosity of the galaxy [4]. Note that to avoid complications with the initial mass function (IMF) of low mass stars, this relation gives the star formation rate for stars with mass $>5 M_{\odot}$. Hence as the total flux density of M82 at $1.4 \mathrm{GHz}$ is $\sim 8.5 \mathrm{Jy}$ and using a distance of $3.2 \mathrm{Mpc}$, then $\operatorname{SFR}\left(M \geq 5 M_{\odot}\right)$ for M82 can be estimated to be $\sim 2.5 \mathrm{M}_{\odot}$ $\mathrm{yr}^{-1}$. Also the radio thermal free-free contribution is reasonably well defined in M82 [2] and can be used to estimate a star formation rate [12] consistent 
with the above value. Finally the star formation rate derived from the total FIR luminosity also gives a value of $\operatorname{SFR}\left(M \geq 5 M_{\odot}\right)$ close to $2 M_{\odot} \mathrm{yr}^{-1}$.

If the SFR is constant over $\sim 10^{7}$ years, then in principle the type II supernova rate can be derived directly from the star formation rate and the initial mass function, on the assumption that all stars with masses $>8 M_{\odot}$ become supernovae. Hence assuming a Miller-Scalo IMF the supernova rate $\nu_{s n}$ is related to the star formation rate via

$$
S F R\left(M \geq 5 M_{\odot}\right)=25 \nu_{s n} \mathrm{M}_{\odot} \mathrm{yr}^{-1} .
$$

Thus, if $S F R\left(M \geq 5 M_{\odot}\right)$ is $\sim 2-2.5 \mathrm{M}_{\odot} \mathrm{yr}^{-1}$ this implies a supernova rate of $\sim 0.08-0.1 \mathrm{yr}^{-1}$ in M82.

Several methods can be used to estimate the supernova rate directly from the radio parameters of the M82 supernova remnants $[10,12]$. The simplest method is to assume that all the M82 remnants which are brighter and smaller than the Cassiopeia A remnant in our galaxy are younger than 330 years. Alternatively if a constant expansion velocity is assumed (e.g., 5000-10000 $\mathrm{km} \mathrm{s}^{-1}$ ) this gives the ages of the remnants and hence the supernova rate [10]. Finally van Buren \& Greenhouse [14] estimated the supernova rates from remnant luminosities. These methods give supernova rates ranging from 0.05 to $0.1 \mathrm{yr}^{-1}$ - consistent with the star formation rates derived above. It is possible that not all supernovae produce radio emitting remnants, and if so this would imply an even larger supernova rates.

However some doubts have been raised concerning the evolution of the remnants in M82. Kronberg et al [7], in a study of the variability of 24 remnants, claim, using statistical arguments, that in a large fraction of the remnants the radio luminosity is decaying at less than $0.1 \%$ per year. If true this could suggest that the remnants are over a thousand years old. Also recent theoretical studies [3] have suggested that the high pressure of the interstellar medium in M82 would result in the supernova remnants expanding at only $500 \mathrm{~km} \mathrm{~s}^{-1}$, hence as the observed sizes are typically a few parsecs, this again would imply ages of thousands of years. Both these results appear to be inconsistent with the supernova rates of $\sim 0.1 \mathrm{yr}^{-1}$ derived above, which require that the ages of typical remnants are a few hundred years.

\section{Interstellar Medium Pressures in M82}

Chevalier \& Fransson [3] assume that the pressure in the starburst region of M82 is $10^{7} \mathrm{~cm}^{-3} K$ and from this derive the low (500 $\left.\mathrm{km} \mathrm{s}^{-1}\right)$ SNR expansion velocities. While there is no doubt that part of the starburst must be at this pressure (e.g., the compact HII regions [9]), it seems likely that much of the starburst is not in static pressure equilibrium and that a range of pressures are present.

As the photoionized component in M82 has a temperature of $\sim 10^{4} \mathrm{~K}$, the pressure assumed by Chevalier \& Fransson would require that the density 

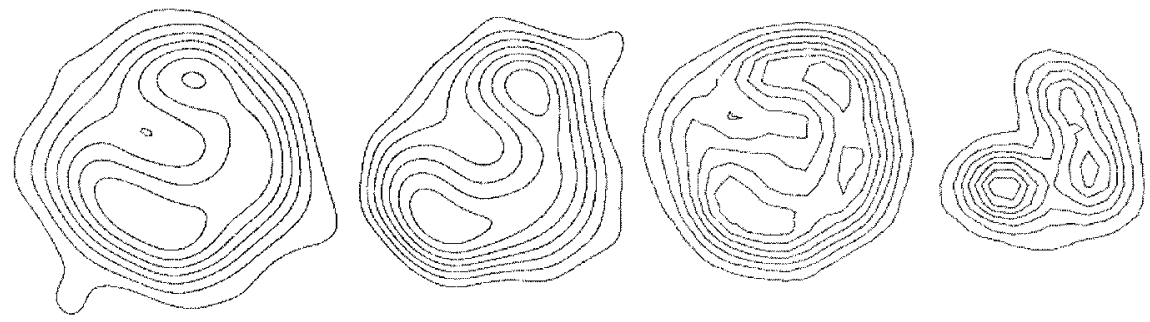

Fig. 2. Four VLBI images ([11] and Riley et al. , this meeting) of the supernova remnant $43.31+59$ taken in $2001.1,1998.9,1997.5$, and 1986.9 convolved to a common angular resolution of 15 mas. The contours levels are at $12.5 \%$ of the peak flux density of each image.

of this gas be $\sim 10^{3} \mathrm{~cm}^{-3}$. However the free-free absorption measurements taken against the individual remnants show emission measures of $\sim 5 \times 10^{5}$ pc $\mathrm{cm}^{-6}[16]$, and hence densities of $\sim 10^{3} \mathrm{~cm}^{-3}$ would imply ionized gas path lengths of only $\sim 0.5 \mathrm{pc}$. Given that the extent of the starburst region is at least $500 \mathrm{pc}$, unless the ionized gas is contrived to occupy the $0.1 \%$ of the volume of the starburst in the immediate vicinity of each remnant, it is difficult to see how the ionized component can provide sufficient pressure to slow the SNR expansion to $500 \mathrm{~km} \mathrm{~s}^{-1}$.

Chevalier\& Fransson suggest that the SNR may be confined by the interclump medium of molecular clouds in M82 and note that many of the remnants are in the line of sight to regions of strong $\mathrm{CO}$ emission. This is, however, not conclusive evidence that the remnants are embedded within the clouds, and often the H1 absorption spectra measured directly against the remnants [17] do not support this interpretation (see discussion of $43.31+592$ below). Furthermore studies by Weiss et al [15] have shown that the molecular gas in the star forming regions of M82 has a kinetic temperatures of $\sim 150 \mathrm{~K}$ and densities of $\sim 10^{3} \mathrm{~cm}^{-3}$ which corresponds to a pressure two orders of magnitude less than that assumed by Chevalier \& Fransson.

Clearly the ISM of M82 is complex, and to assign a single pressure to the starburst region may be unrealistic. Hence it is likely that many of the SNR in M82 are embedded in regions with pressures significantly lower than $10^{7}$ $\mathrm{cm}^{-3} \mathrm{~K}$ and hence have expansion velocities $>500 \mathrm{~km} \mathrm{~s}^{-1}$.

\subsection{Measuring Expansion Velocities}

Now that we can resolve the compact sources in M82 [10], in principle it is a relatively simple matter to measure their expansion velocities by measuring their size over a period of time, although such studies are limited by current instrumental sensitivity to the brightest sources. The first expansion measurements were made by Pedlar et al [11] on the compact sources $41.95+575$ $\& 43.31+592$. The brightest, most compact source $(41.95+575)$ was shown to 


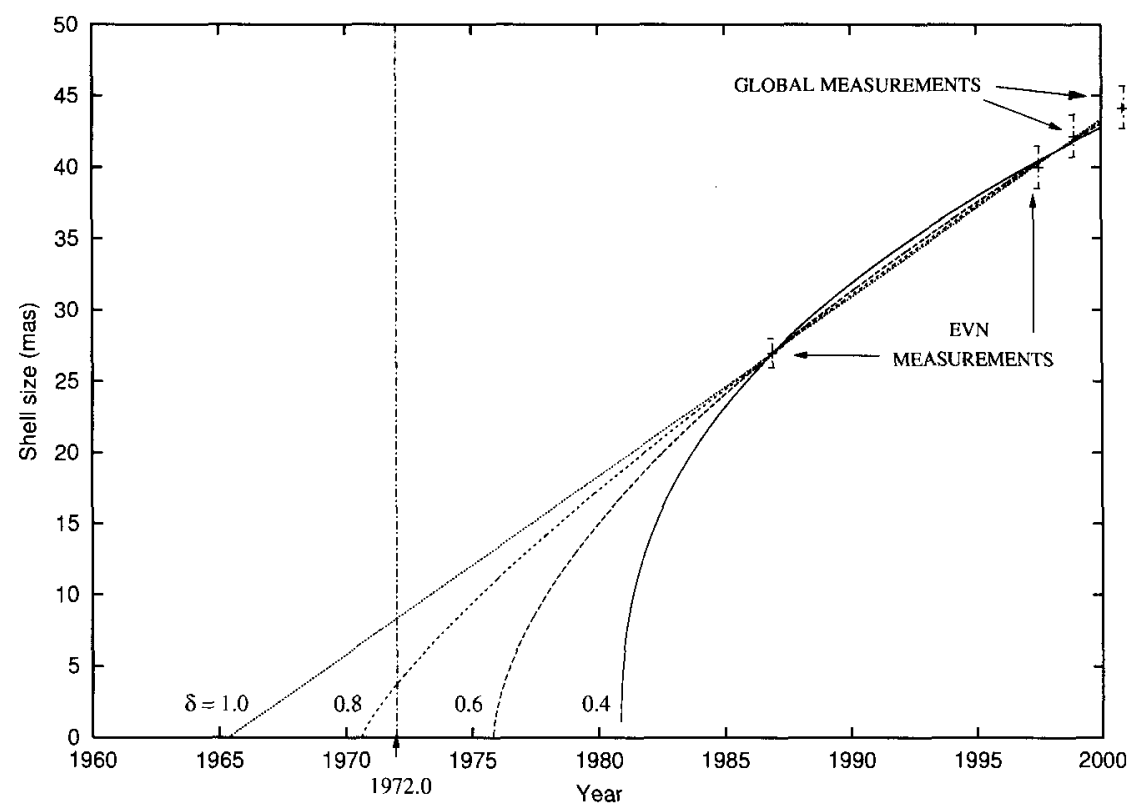

Fig. 3. The size of $43.31+592$ plotted as a function of age including the 2001.1 measurement. The Figure also shows different expansion models. Adapted from McDonald et al [8].

have an elongated structure and the expansion velocity along the major axis was constrained to $<4000 \mathrm{~km} \mathrm{~s}^{-1}$. Subsequent global VLBI measurements [8] showed it to have an expansion velocity of $\sim 2000 \mathrm{~km} \mathrm{~s}^{-1}$. This source is, however, rather anomalous on account of its high radio luminosity and its rapid decay. Also the global VLBI structure is highly elongated and more like a bipolar structure than a shell - unlike a typical SNR [8].

The source $43.31+592$ is much more typical an SNR of shows a welldefined shell structure (Fig. 2). By comparing data observed in 1986 and 1997, Pedlar et al [11] deduced an expansion velocity of $\sim 10000 \mathrm{~km} \mathrm{~s}^{-1}$. Subsequent Global VLBI measurements ([8] and Riley et al. , this meeting) have confirmed this result and shown its expansion to be consistent with no deceleration (Fig. 3), implying an age of about 35 years. As the SNR seems to be in free expansion we can set a simple constraint of $<2000$ atoms cm $\mathrm{cm}^{-3}$ to the external density by assuming that the mass of gas swept up is less than the mass ejected. The expansion velocity of this source clearly exceeds the $500 \mathrm{~km} \mathrm{~s}^{-1}$ predicted by Chevalier \& Fransson, which may be accounted for if it is in a region with relatively low external pressure. This assertion is supported by the lack of a low frequency turnover at $73 \mathrm{~cm} \mathrm{[16]} \mathrm{in} \mathrm{the}$ spectrum of $43.31+592$ even though it is in the line of sight to regions with strong free-free emission. This shows that the remnant is in front of, and 
not embedded in, the relatively dense ionized gas in the central region of M82. Even though relatively strong $\mathrm{H} 1$ absorption $\left(5 \times 10^{21}\right.$ atoms $\left.\mathrm{cm}^{-2}\right)$ is seen against this remnant [17], its narrow width and systemic velocity is consistent with absorption by a disk of $\mathrm{H} 1$ external to the central region of M82. Hence this is further evidence that this SNR is not embedded in neutral gas the center of the starburst. Thus if this SNR is in a relatively low pressure region, it may be possibly reconcile the difference between the 500 $\mathrm{km} \mathrm{s}^{-1}$ predicted by Chevalier \& Fransson and the measured $\sim 10000 \mathrm{~km} \mathrm{~s}^{-1}$ expansion velocity.

Recent MERLIN observations (Muxlow et al. , this meeting) have independently confirmed the above expansion velocities and also measured the expansion velocities of 2 more remnants. One of the remnants has a velocity close to $10000 \mathrm{~km} \mathrm{~s}^{-1}$, whereas the other is relatively low at $2000 \mathrm{~km}$ $\mathrm{s}^{-1}$. We now have 4 expansion measurements and find little evidence for 500 $\mathrm{km} \mathrm{s}^{-1}$ expansion velocities. Hence we see no reason to revise the supernova rates or remnant ages that we have inferred previously. Higher sensitivity observations using e-MERLIN, combined with an increasing time baseline, will enable the expansion velocities of many more remnants in M82 to be determined and hence constrain both the supernova rate and the parameters of the interstellar medium in M82.

\section{References}

1. M.L. Allen, P.P. Kronberg: Astrophys. J. 502, 218 (1998)

2. J.E. Carlstrom, P.P. Kronberg: Astrophys. J. 366, 422 (1991)

3. R.A. Chevalier, C. Fransson: Astrophys. J. 558, 27 (2001)

4. L. Cram et al. : Astrophys. J. 507, 155 (1998)

5. P.P. Kronberg, P.N. Wilkinson: Astrophys. J. 200, 430 (1975)

6. P.P. Kronberg, P. Biermann, F.R. Schwab: Astrophys. J. 291, 693 (1985)

7. P.P. Kronberg et al. : Astrophys. J. 535, 706 (2000)

8. A.R. McDonald et al. : Mon. Not. R. Astron. Soc. 322, 100 (2001)

9. A.R. McDonald et al. : Mon. Not. R. Astron. Soc. 334, 912 (2002)

10. T.W.B. Muxlow et al. : Mon. Not. R. Astron. Soc. 266, 455 (1994)

11. A. Pedlar et al. : Mon. Not. R. Astron. Soc. 307, 761 (1999)

12. A.Pedlar: "Extragalactic supernovae and the starformation rate." In: Proc. IAU symposium 205, ed. by R. Schilizzi (ASP 2001) p. 366

13. S.W. Unger et al. : Mon. Not. R. Astron. Soc. 211, 783 (1984)

14. D. van Buren, M.A. Greenhouse: Astrophys. J. 431, 640 (1994)

15. A. Weiss et al. : Astron. Astrophys. 365, 571 (2001)

16. K.A. Wills et al. : Mon. Not. R. Astron. Soc. 291, 517 (1997)

17. K.A. Wills, A. Pedlar, T.W.B. Muxlow: Mon. Not. R. Astron. Soc. 298, 347 (1998) 